\title{
S-DOMAIN METHODOLOGY FOR ASSESSING THE SMALL SIGNAL STABILITY OF COMPLEX SYSTEMS IN NON-SINUSOIDAL STEADY STATE
}

\author{
Adam Semlyen \\ Life Fellow, IEEE \\ Department of Electrical and Computer Engineering \\ University of Toronto \\ Toronto, Ontario, Canada M5S 3G4
}

\begin{abstract}
The paper gives the cutline of a generalized small signal stability analysis of the periodic steady state, which includes harmonics, for systems with complex components. These could be (switched) time-varying, or nonlinear components, possibly also rotating machines and transmission lines represented as elements with distributed and frequency-dependent parameters. It is assumed that a Harmonic Power Flow solution exists. Its stability analysis by linearization along the known limit cycle would be computationally too expensive. However, linearization of the individual components results in linear, time-periodic (LTP) models. The proposed solution is based on equivalencing the LTP models with standard linear timeinvariant (LTI) components The methodology is illustrated on a small system with a periodic steady state that may be either stable or unstable.
\end{abstract}

\section{INTRODUCTION}

Many of today's power systems include elements that are not constant and linear. These are actual nonlinear elements (such as arc furnaces or magnetizing branches of transformers) and timevarying components (converters and power semiconductor devices). Their effect on the power system in its steady state operation is the appearance of harmonics. In their presence, the distributed nature and frequency-dependence of transmission lines also acquires significance, in addition to the lumped parameter, linear, time-invariant (LTI) elements. Also rotating machines have to be represented, for accurate modeling, in phase coordinates: in this case the inductance matrix of the machine is itself time-varying [1]. Under these circumstances, the steady state condition of the power system is readily obtained by one of a variety of so-called Harmonic Power Flow programs [2-13].

Just as in the case of the steady state condition of a power system calculated by a conventional Power Flow program, the harmonic steady state could also be stable or unstable, and thus a problem of small signal stability analysis exists and may require solution. Unless the steady state has been obtained by timedomain simulation, the solution may be unstable. One of the causes could be the effect of negative slip (resulting in a negative resistance) in rotating machines in relation to a transient rotating field, even if (for example in an induction motor) the steady state slip is non-negative. Stability analysis reported in the literature has focused on individual components, of a particular type [1415]. Since, however, stability is a system-wide problem, the purpose of this paper is to provide an outline of a generalized small signal stability assessment methodology of the periodic steady state (which contains harmonics) for complete systems that include frequency-dependent, time-varying, or nonlinear components. It addresses the problem of identifying the critical modes in the transient of a perturbed Harmonic Power Flow solution.

PE-754-PWRS-2-3-1998 A paper recommended and approved by the IEEE Power System Analysis, Computing and Economics Committee of the IEEE Power Engineering Society for publication in the IEEE Transactions on Power Systems. Manuscript submitted December 11 1996; made available for printing March 9, 1998.
Fundamentally, a transient mode in a constant parameter linear system may be given by an $s$-domain (i.e. complex frequency) solution. In the more general case examined here, the solution, while still in the frequency-domain, is slightly more complex. The problem consists in examining a transient around a periodic steady state solution obtained by the Harmonic Power Flow calculation. Since this "variational" problem is linear, it can be easily reformulated in the complex frequency or $s$-domain. The key to achieving this consists in substituting for the linear, time-periodic (LTP) elements, obtained by linearization, $\underline{L T I}$ equivalents, i.e. linear time-invariant components. Thus, the frequency-domain problem formulation, which is natural for components with frequency-dependent parameters (transmission lines) and intrinsic to eigenvalue analysis, can be fully and conveniently applied to the generalized small signal stability analysis problem.

The Harmonic Power Flow corresponds to a periodic solution of the nonlinear ODEs (ordinary differential equations) of the complete system. Linearization of the ODEs in the neighborhood of the solution yields the linear, homogeneous equation

$$
\dot{x}=A(t) x
$$

where $A(t)$ is a real $T$-periodic coefficient matrix. By classical Floquet theory [16-18], the general solution of (1) is of the form: $x(t)=R(t) e^{B t} x(0)$, where $B$ is a constant matrix and $R(t)$ a $T$. periodic matrix. Unfortunately, the formulation of (1) and the computation of the matrices $B$ and $R(t)$ is in general a very difficult task and will therefore not be addressed directly in this paper. However, the solution for any mode of (1) (see [17] p.56) has the simple form

$$
e^{s t} r(t)
$$

where $s$ is a (generally complex) eigenvalue of $B$ and $r(t)$ is a $T$-periodic vector. This fact will be exploited in our study.

The essential difference between a mode given by (2) and a mode in the case $A=$ const is that in the latter case the periodic multiplier $r(t)$ is absent. While even the imaginary part of $s$, if it exists, is unrelated to $T, r(t)$ contains the base frequency $\omega=2 \pi / T$ and its harmonics and consequently does not affect the value of the transient mode (2) at the discrete periodic points $t=T$. Therefore, the exponential factor $e^{s t}$ of (2) can be considered the essential part for assessing the stability of the mode, while the periodic factor $r(t)$ has merely secondary significance by producing only a modulation of the transient mode (2) between the discrete T-periodic samples. This remark is the basic underlying idea for the above mentioned equivalencing procedure of LTP components by LTI elements on which the methodology of this paper is based.

The content of the main body of the paper is as follows:

In Section 2 the $s$-domain methodology is first presented in general terms. Next, details are given describing the derivation of the LTI-equivalents and the modeling of other components.

In Section 3 an illustrative example is given with results both in the $s$-domain and through time-domain simulations. 


\section{S-DOMAIN METHODOLOGY}

2.1 Overview of Methodology

In the following it is assumed that both a Harmonic Power Flow solution and its linearization (along the periodic steady state trajectory) already exist. However, the alternative of its direct stability analysis, using Floquet theory, would be computationally too expensive for nontrivial three-phase systems, because of the huge size of the systern matrix $A(t)$ and the need for its calculation at a fairly large number of time samples $t$ within a period $T$. Moreover, the network itself, having distributed parameters resulting in frequency-dependence, does not readily lend itself to state equation representation. Therefore, in the computational procedure of this paper a system matrix is built from its components and the time variation within a period is only implicitly taken into account. The general methodology can be described by the following steps.

a. Lumped parameter, L.TI elements are described in standard state variable form by

$$
\begin{aligned}
& \dot{x}=A x+B u \\
& y=C x+D u
\end{aligned}
$$

It is assumed that $u$ is a vector of input voltages and $y$ is a vector of terminal currents. In the $s$-domain the corresponding relations are

$$
\begin{gathered}
y=(s I-A)^{-1} B u \\
y=H(s) u
\end{gathered}
$$

$$
H(s)=C(s I-A)^{-1} B+D
$$

In the case of distributed (possibly, frequency-dependent) parameter, i.e., infinite dimensional components, an input/output relation of the form (5) still exists but the matrix transfer function $H(s)$ is no longer rational as in (6) (it has transcendental elements, such as hyperbolic functions in $s$ ).

The linearized, variational equations of nonlinear and timevarying (periodically switched) components are linear, timeperiodic (LTP):

$$
\begin{aligned}
& \dot{x}=A_{t} x+B_{t} u \\
& y=C_{t} x+D_{t} u
\end{aligned}
$$

Here $A_{t}, B_{t}, C_{t}, D_{t}$ are $T$-periodic functions of time. If we focus on a single mode $e^{s t}$ of a transient in the complete system, then this exponential variation applies for all state variables and algebraically related other variables, such as $u$ and $y$. In Section 2.2 it will be shown that under this assumption of exponential pattern $e^{s t}$ of variation, the LTP equations (7) can be replaced by equivalent LTI equations (3) and a corresponding input/output equation (5). This is an $s, T$-equivalent, meaning that it is valid for the mode $e^{s t}$ and produces the same results as the LTP equations (7) at periodic intervals $T$ but not in-between. It is thus an $s, T$-stability equivalent.

b. Assemble all input/output relations (5) into a single nodal equation for the whole system, with the currents in each node adding to zero. If $v$ denotes the union of all nodal voltages $u$, the resultant equation is

$$
Y(s) v=0
$$

where $Y(s)$ is an $s$-domain admittance matrix. Clearly, (8) has a non-trivial solution for $v$ only if $Y(s)$ is singular. This (usual) argument indicates that $s$ is an eigenvalue of $Y(s)$ but not in the sense of customary, constant matrices, that would lead to the eigenvalue problem

$$
(x \mid I-Y(s)) v=0
$$

For (9) to have a non-trivial solution for $v$, we must have

$$
\lambda_{\text {tiin }}=f(s) \rightarrow 0
$$

This suggests that obtaining an eigenvalue $s$, defined as a root of $\operatorname{det}(Y(s))=0$, could be achieved by iteratively solving $(10)$ for $s$. c. The solution of (10) can be achieved easily using the Secant Method. In our implementation it involves the following steps:

- Starting values for $s$ are chosen as points $\mathrm{j} \omega_{k}$ along the imaginary axis because of its significance in stability assessment. A second value (needed in the secant algorithm) can be chosen nearby.

- For a given $s, Y(s)$ is calculated, then $\lambda_{\min }$, its smallest eigenvalue (in absolute value). In small problems a QR algorithm can be used, and in large problems the inverse power method is appropriate for this purpose.

- With two given values of $s$, the secant method gives the new value

$$
s^{k+2}=\frac{s^{k} \lambda_{\min }^{k+1}-s^{k+1} \lambda_{\min }^{k}}{\lambda_{\min }^{k+1}-\lambda_{\min }^{k}}
$$

Stability of the system is concluded if all converged values $s$ have negative real part.

\subsection{Linear Periodic Systems}

\subsubsection{Solution of Linear Time-Varying ODEs}

The solution of the linear, time-varying ODE (7a) with the initial condition $x(0)=x_{o}$, in the general case when $A_{t}, B_{t}$ are not necessarily periodic, can be obtained using the transformation

$$
\begin{gathered}
x=E_{t} z \\
E(0)=I, \quad z(0)=x_{o}
\end{gathered}
$$

Substitution of (12a) into (7a) gives

$$
\dot{E}_{t} z+E_{t} \dot{z}=A_{t} E_{t} z+B_{t} u
$$

If we impose on $E_{t}$ the condition

$$
\dot{E}_{t}=A_{t} E_{t}
$$

(such that it becomes a basic solution matrix for the homogeneous part of (7a)) then (13) reduces to

$$
E_{t} \dot{z}=B_{t} u
$$

Integration of $\dot{z}$ from (13b) with the initial condition (12b) and substitution into (12a) yields the final result

$$
x_{t}=E_{t} x_{o}+E_{t} \int_{0}^{t} E_{\tau}^{-1} B_{\tau} u_{\tau} d \tau
$$

For numerical calculations, $E_{t}$ and $E_{\tau}$ used in (14) can be easily obtained by integrating (13a) with initial condition (12b), using for instance the trapezoidal rule with $A_{t}$ evaluated at mid time step.

In the particular case (3a) of an LTI component, we have

$$
E_{t}=e^{A t}
$$

and (14) gives the familiar expression

$$
x_{t}=e^{A t} x_{o}+\int_{0}^{t} e^{A(t-\tau)} B u(\tau) d \tau
$$

\section{2 .2 Identification of $L T I$ Equivalents}

Next we focus on a particular mode so that

$$
\begin{aligned}
x_{T} & =e^{s T} x_{o} \\
u_{t} & =e^{s t} u_{o}
\end{aligned}
$$

Substitution of (17b) into (14) gives

$$
x_{i}=E_{t} x_{o}+E_{t}\left(\int_{0}^{t} E_{\tau}^{-1} B_{\tau} e^{s \tau} d \tau\right) u_{o}=E_{t} x_{o}+F_{t}(s) u_{o}
$$

where $F_{t}(s)$ is obtained using numerical integration. For $t=T$, and taking (17a) into account, (18) gives the simple relation

$$
e^{s T} x=E x+F(s) u
$$


or

$$
x=\left(e^{s T} I-E\right)^{-1} F(s) u
$$

At this point we note that from (15)

$$
A=\frac{1}{T} \log (E)
$$

Comparing (19b) with (4) we get

$$
B=(s I-A)\left(e^{s T} I-E\right)^{-1} F(s)
$$

Thus, we have obtained in (20) the coefficients $A$ and $B$ of an LTI equivalent (3) for a given LTP component. It is valid for the complex frequency $s$ and periods $T$. The coefficients $C$ and $D$ of (3b) are simply the values of $C_{t}$ and $D_{t}$ from (7b) for $t=0$.

\subsection{Other Types of Components}

\subsubsection{Components with Distributed and Frequency-Dependen Parameters}

While the admittances $Y(s)$ of lumped components are obtained simply from $R, s L$, and $s C$ (and the representation of any other lumped, possibly control related, element is obtained in a similar way based on (3)), in the case of a transmission line we use the sequence of expressions:

$Z=R+s L, Y=s C, \Gamma=\sqrt{Z Y}, Y_{c}=\sqrt{Y / Z}, Y_{i n}(s)=Y_{c} / \tanh (\Gamma)(21)$

These have been written, for simplicity, for the single phase (or single mode) case. In (21) the input admittance $Y_{i n}(s)$ of the line corresponds to a single end (the other end where a voltage source might be connected appears as a short-circuit in small signal stability calculation). Clearly, $Y_{i n}(s)$ is a complex, but calculable, function of the complex frequency $s$.

In order to include frequency dependence in $R$ and $L$ in (21), it is convenient to use the familiar analytical expression

$$
p=\frac{1}{\sqrt{s \mu_{o} \sigma}}
$$

for the complex penetration depth, for both conductor and earth In the case of the conductor resistance, we use the low and high frequency formulae [19]

$$
R_{o}=\frac{l}{\pi r^{2} \sigma}, \quad Z_{\infty}=\frac{l}{2 \pi r \sigma p}
$$

and combine them to give

$$
Z(s)=\sqrt{R_{o}^{2}+Z_{\infty}^{2}}=k(s) R_{o} ; \quad k(s)=\sqrt{1+\left(\frac{r}{2 p}\right)^{2}}
$$

The expression (23b) of $Z(s)$ is asymptotically accurate at both low and high frequencies and even in-between the error remains below 7\% (with respect to analytical formulae based on Bessel functions).

Similarly, as outlined in [19] and [20], the use of the complex depth $p$ of (22) results in the function $L(s)$ of $s$ to be used in (21).

\subsubsection{Rotating Machines and Negative Damping of Transients}

Rotating machines may have a crucial effect on small signal stability due to an implicit "induction machine effect" even if the machine is a synchronous generator. This fact is not readily apparent from the complex set of general machine equations [1], but it is well known and clearly reflected by the equivalent resistance $R / S$ ( $S=$ slip, as opposed to $s=$ complex frequency) which becomes negative if the slip is negative and, combined with the resistance of the system, may result in overall negative damping. We reiterate however that, from the point of view of a transient, the slip $S$ should be related to $s$ rather than to the steady state synchronous frequency $\omega$. Consequently, because of the fundamental significance of the induction machine effect, we shall focus in the following on the corresponding equations.

Figure 1 represents a simplified one-line diagram for the stator-rotor relation of an induction machine. The variables $v, i, \Phi$ are in the stator frame of reference, while $v_{r}, i_{r}, \Phi_{r}$ are in the rotor frame of reference. Their relation is given by the factor $\exp \left(\mathrm{j} \omega_{r} t\right)$ where $\omega_{r}$ is the rotor speed:

$$
\begin{aligned}
i_{r} & =e^{-\mathrm{j} \omega_{r} t_{i}} \\
\Phi_{r} & =e^{-\mathrm{j} \omega_{r} t} \Phi
\end{aligned}
$$

(no similar equation applies for $v$ and $v_{r}$ ).

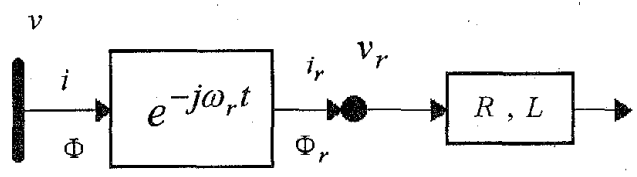

Fig. I Simplified representation of induction machine

We note that the instantaneous variables in this section $\left(v, i, \Phi, v_{r}, i_{r}, \Phi_{r}\right)$ are conveniently represented as complex since then their real and imaginary components can be viewed as corresponding to two-phase coordinates (in sinusoidal steady state, to $\cos \omega t$ in one phase corresponds $\sin \omega t$ in the other phase) and (24) gives the coordinate transformations. This representation is also convenient for the time-domain simulations in Section 3.4 .

The corresponding ODEs are

$$
\begin{gathered}
\dot{\Phi}=v \\
\dot{\Phi}_{r}=v_{r} \\
i_{r}=-\frac{R}{L} i_{r}+\frac{1}{L} v_{r}
\end{gathered}
$$

By taking the derivatives of (24) we get

$$
\begin{aligned}
i_{r} & =e^{-\mathrm{j} \omega_{r} t}\left(\dot{i}-\mathrm{j} \omega_{r} i\right) \\
\dot{\Phi}_{r} & =e^{-\mathrm{j} \omega_{r} t}\left(\dot{\Phi}-\mathrm{j} \omega_{r} \Phi\right)
\end{aligned}
$$

Substitution of (25a) and (25b) into (26b) gives

$$
v_{r}=e^{-\mathrm{j} \omega_{r} t}\left(v-\mathrm{j} \omega_{r} \Phi\right)
$$

Substitution of (24a), (26a), and (27) into (25c) gives

$$
i=\left(\mathrm{j} \omega_{r}-\frac{R}{L}\right) i+\frac{1}{L}\left(v-\mathrm{j} \omega_{r} \Phi\right)
$$

to which we append (25a):

$$
\dot{\Phi}=v
$$

Equations (28) are the simplified state equations of the induction machine. Note, that $\Phi$ is also a state variable.

The $s$-domain equivalent of (28) is obtained by the substitution $\frac{d}{d t} \rightarrow s$ and elimination of $\Phi$. This yields

$$
i=\frac{v}{\frac{R}{S}+s L}
$$

where $S$ is the $s$-domain slip:

$$
S=\frac{s-\mathbf{j} \omega_{r}}{s}
$$

Since for the steady state case, i.e. when $s=\mathrm{j} \omega,(30)$ gives the traditional definition of the slip, equation (29) is in full accordance with our expectations. 


\section{ILLUSTRATIVE EXAMPLE}

\subsection{The Test System}

The test system has been selected to include, in addition to lumped and distributel parameter elements, a nonlinear component and an induction motor; see Figure 2. While the system appears in steady state to be passive and therefore stable, the induction motor $\mathrm{M}$ may have at some complex frequency $s$ a slip $S$ (see (30)) with regative real part so that the resulting negative resistance may render unstable the periodic steady state. This will be particularly the case if the rating of the motor $P_{\text {motor }}$ is small, since then $R$ in (29) is large.

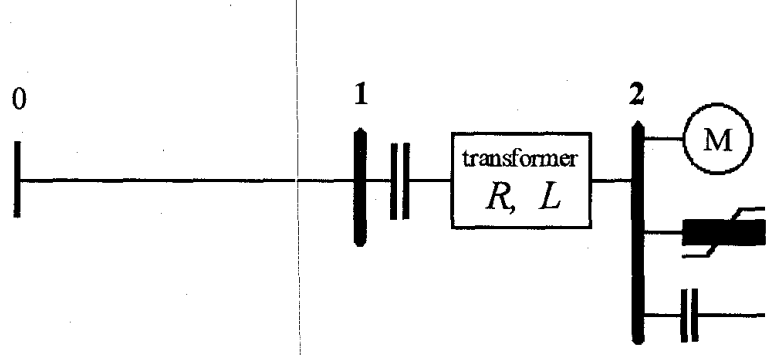

Fig. 2 Study system

All data have beer specified in per unit, except for $\omega=377 \mathrm{rad} / \mathrm{s}$. In the rotor, $\omega_{r}=0.98 \omega, R=0.02, \omega L=0.3$. The nonlinear branch has $R=0.01$, and its inductance is described by

$$
i=\alpha \psi+\beta \psi^{5}
$$

with $\alpha=0.01, \beta=0.1$. The capacitance of the load bus 2 is given by $\omega C=0.05$. Between nodes 1 and 2 there is a series capacitance given by $\omega C=5$ and a transformer represented by $R=0.01, \omega L=0.1$. The source bus has been denoted by 0 since in an incremental sense it has zero voltage. The line between nodes 0 and 1 is assumed to have distributed, balanced linemode, parameters with the total values over its whole length of $R=0.05, \omega L=0.3$, and $\omega C=0.3$. The conductor impedance was modeled as frequency-dependent, as shown in (23b).

Figure 2 indicates that the admittance matrix for this system relates to nodes 1 and 2 and is thus of dimension $2 \times 2$.

\subsection{Periodic Steady State}

In order to simplify this part of the computations, it was assumed that in (31)

$$
\begin{aligned}
& \text { Then (31) becomes } \\
& i=\left(\alpha+\frac{5}{8} \beta\right) \cos \omega t+\frac{5}{16} \beta \cos 3 \omega t+\frac{1}{16} \beta \cos 5 \omega t
\end{aligned}
$$

and, with the inductor voltage $\dot{\psi}$ from (32), all voltages and currents of the periodic steady state of Figure 2 can be calculated. Most of these are of course irrelevant for the subsequent variational calculations bit the linearization of (31) around the periodic steady state (32) is needed:

$$
\Delta i=\left(\alpha+5 \beta \psi^{4}\right) \Delta \psi=\left(\alpha+5 \beta\left(\frac{3}{8}+\frac{1}{2} \cos 2 \omega t+\frac{1}{8} \cos 4 \omega t\right)\right) \Delta \psi
$$

With (34) the (variational) LTP differential equation of the nonlinear branch

$$
\begin{gathered}
\Delta \dot{\psi}=-R \Delta i+\Delta v \\
\text { becomes } \\
\Delta \dot{\psi}=-R\left(\alpha+5 \beta\left(\frac{3}{8}+\frac{1}{2} \cos 2 \omega t+\frac{1}{8} \cos 4 \omega t\right)\right) \Delta \psi+\Delta v
\end{gathered}
$$

This equation is of form ( 7 a) so that the equivalencing procedure of Section 2.2 can directly be used. For the output relation (7b) at $t=0$ we use (34), i.e.,

$$
\Delta i=(\alpha+5 \beta) \Delta \psi
$$

We have thus in (36) the (variational) LTP differential equations (7) for the nonlinear branch of Figure 2.

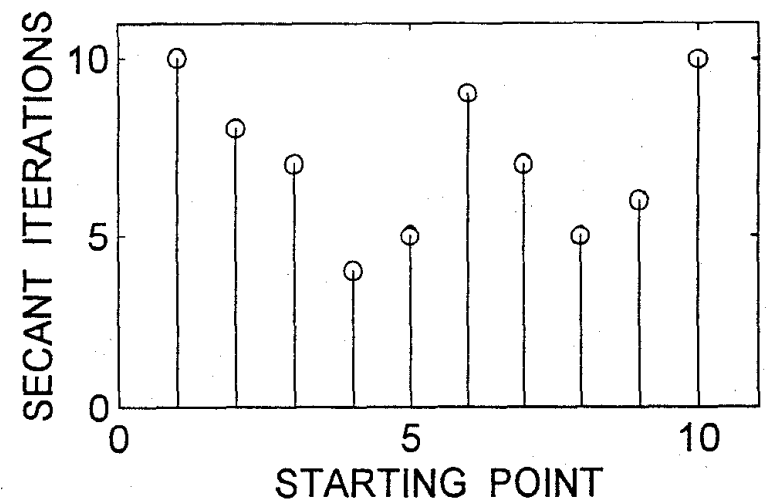

Fig. 3 Number of secant iterations for different starting points
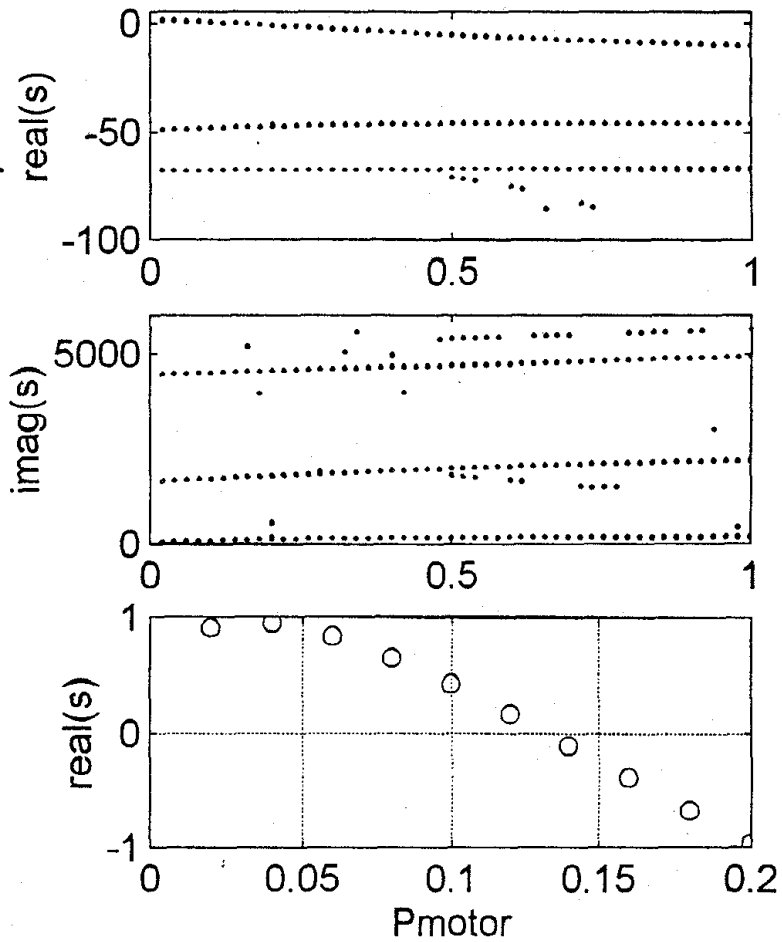

Fig. 4 Values of $s$ function of $P_{\text {motor }}$

(The last plot zooms in to the critical part of the first plot)

\section{$3.3 s$-Domain Results}

The s-domain computations start with a selection of initial values for $s$ on the imaginary axis. Then the methodology of Section 2 is applied. In the following example, 10 logarithmically placed initial values are used, with the imaginary parts: $\omega_{k}=10 \times 2^{k-1} \quad(k=1,2, \ldots 10)$. Figure 3 shows the convergence of the secant iterations (11) to a tolerance of $10^{-12}$, with $P_{\text {motor }}=0.1$.

Three values of $s$ have been reached from the 10 starting points of Figure 3:

$$
\begin{aligned}
& \text { from } k=1-5 \rightarrow s=0.4158+\mathrm{j} 89.603 \\
& \text { from } k=6-9 \rightarrow s=-48.024+\mathrm{j} 1717.7
\end{aligned}
$$$$
\text { from } k=10 \rightarrow s=-67.677+4501.8
$$

It is interesting to note that the first $s(=0.4158+\mathrm{j} 89.603$ in (37a)) is unstable and it is reached fastest (in only 4 iterations) from the starting point $k=4\left(\omega_{4}=80\right)$ of Figure 3 , to which it is 
closest. Not surprisingly, this $s$ is subsynchronous and the corresponding slip (real part of $S$ obtained from (30)) is negative $(=-3.123)$.

A similar remark applies to $s=-48.024+\mathrm{j} 1717.7$ in (37b) which, however, is stable and supersynchronous. At higher values of $k$, the results are likely to be less accurate and reliable because of the errors involved in the numerical integrations. Therefore, these are not shown on the next plots (Figure 4).

Since the resistance of the induction motor decreases with its power rating, the system is expected to become more stable for larger $P_{\text {motor }}$. Figure 4 represents the real and imaginary parts of $s$ for $P_{\text {motor }}$ varied in small steps ( 0.02 p.u.). As shown above in (37) for specific cases, larger values of $\operatorname{Im}(s)$ correspond to larger values of $\operatorname{Re}(s)$ in the first two plots of Figure 4 . The only part where $\operatorname{Re}(s)$ indicates instability is in the upper left corner of the top subplot in Figure 4. A close-up of it is shown in the bottom subplot. It shows clearly that, as expected, the system becomes increasingly unstable with decreasing $P_{\text {motor }}$ (below $P_{\text {motor }}=0.13$ ). Since a positive $\operatorname{Re}(s)$ is less than 1 , the time constant of an unstable transient is larger than 1 second, possibly by a large factor. This affects the requirements for the timedomain simulations to be discussed next.

\subsection{Time-domain Verification}

Simulations with the purpose of demonstrating the stability or instability of the test system will have to extend over dozens or even hundreds of $(60 \mathrm{~Hz})$ periods. The time step must be small enough to assure good resolution within each period. In addition, the system of ODEs involved is stiff, especially if the transmission line is modeled by a larger number of lumped parameters. This would lead to even smaller time steps. (Modeling of frequency dependence was not even considered.) To keep the simulation time acceptable, the time-domain simulation methodology (for this nonlinear problem) had to be chosen with great care. A quasi-trapezoidal hybrid approach was therefore used to assure stable and accurate simulation with maximal time steps. Its details are, however, trivial in the context of this study.

Figure 5 gives the result of two simulations. For clarity, only the peaks (at both polarities) of the oscillatory voltage at the load bus are shown. At the top, $P_{\text {motor }}=0.5$ and the system is stable, as expected. A number of 48 periods were more than sufficient to demonstrate it. In the bottom subplot, $P_{\text {motor }}=0.1$, the system is unstable and ten times as many periods were used to show it clearly. Because of error accumulation in the numerical integration, it is not expected that the instability in this plot exactly reflects the value of $\operatorname{Re}(s)$ in $(37 a)$.
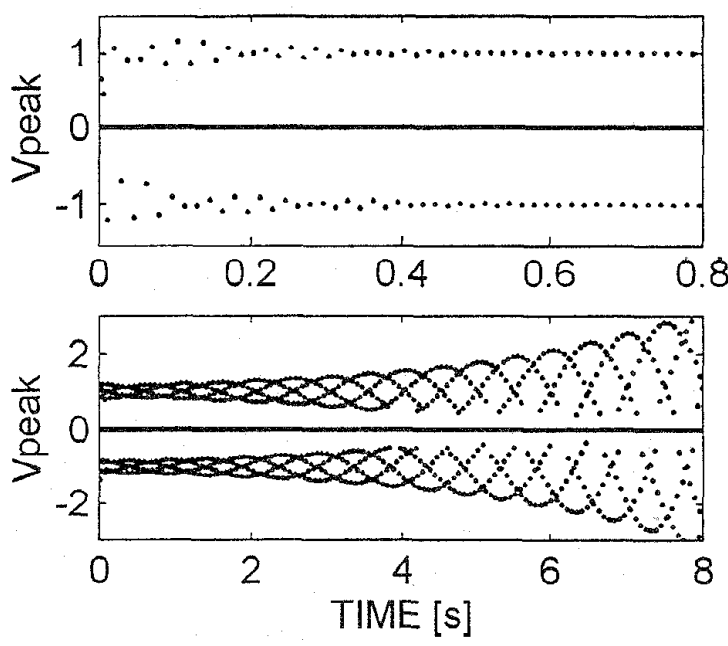

Fig. 5 Peak values of voltage at load bus 2 of Fig. 2

Top: $P_{\text {motor }}=0.5$ (stable); Bottom: $P_{\text {motor }}=0.1$ (unstable)

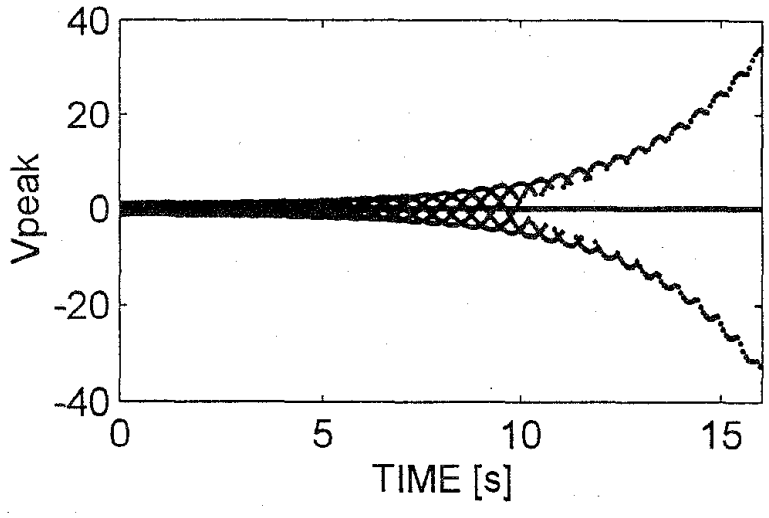

Fig. 6 Peak values of voltage over longer time interval
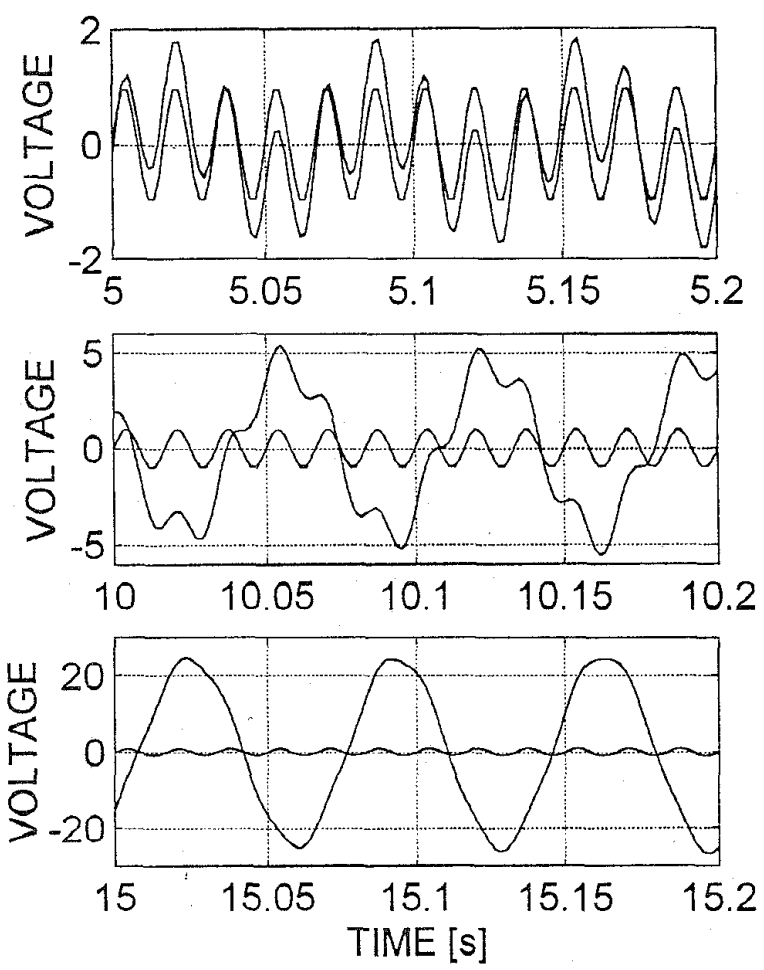

Fig. 7 Portions of the transient at three stages (A second trace in each subplot gives $\sin \omega t$ for reference)

The intricate pattern of the peak values displayed in (the bottom) Figure 5 suggests the presence of more than one frequeney somponent in the yoltage transient. It would be expected that the oscillatory part of $s$, given by $\operatorname{Im}(s)=\mathrm{j} 89.603$ in (37a), which is by a factor of $\sim 4.2$ slower than the base periodic steady state voltage $\sin \omega t$ (with $\omega=377$ ), is reflected in the interference pattern of Figure 5.

To show this, the plot of (the bottom) Figure 5 was extended to a double time interval in Figure 6, so as to render the transient part, $\operatorname{Im}(s)=\mathrm{j} 89.603$, dominant over the steady state component sin $\omega t$. Consequently, the interference pattern gradually vanishes.

A closer look, given in Figure 7, at portions of the transient for which only the peak values are displayed in Figure 6, permits to demonstrate that the time-domain simulations correctly reflect not only the (negative) damping effect given by $\operatorname{Re}(s)$ of (37a) but also the oscillatory effect, close to a subharmonic of order 4 , due 
to $\operatorname{Im}(s)$ of $(37 \mathrm{a})$. The three parts of Figure 7 zoom in to short time intervals ( 0.2 seconds each, only 12 periods), at an early, a middle, and an advanced stage of the transient. For reference, each subplot contains the approximate steady state value $\sin \omega t$, in addition to the plot of the transient. In the upper Figure 7, the transient departs by less than 1 p.u. from the sinusoidal base, but it can be seen that the superimposed oscillations have an approximately 4 times larger period. In the middle Figure 7 , the subharmonic is more prominently visible and we can see that it is not an integer (true) subharmonic since the shape of the resultant transient gradually changes. In the bottom Figure 7 , practically only the "subharmonic" remains and we see that there are slightly less than three (subharmcnic) periods over 12 base frequency periods. This is in very good accordance with the ratio of 4.2 established above, based on the $s$-domain result (37a).

\subsection{Overview of Results}

The example chosen for this study has included the most representative elements of a power system. The modeling of a time-varying (switched) component will be separately illustrated in the next section. In fact, in the linearized analysis of this study, both time-varying and nonlinear components become linear timeperiodic (LTP) elements for which linear time-invariant (LTI) equivalents have been successfully used.

The $s$-domain approach is fast and accurate. For the small system used, the converged values of $s$ have been obtained (for a fixed $P_{\text {motor }}$ ) in about ten seconds (with MATLAB v. $4.2 \mathrm{c}$ ), while time-domain simulations look several hours in some cases. Moreover, time-domain results are less reliable and more difficult to interpret than values obtlined for the complex frequency $s$.

The illustration presented was, for good reasons, very simple, compared to the complexity of real systems. (This permitted, for example, the computation of eigenvalues by direct, rather than iterative, methods.) Section 3.7 gives a preview of how a realistic, large scale study could be performed using the proposed $s$-domain methodology.

\subsection{Mcideling of a TCSC}

The example of a Thyristor Controlled Series Capacitor (TCSC) is used in the following to illustrate the modeling of a time-varying component.

The TCSC consists of a capacitor $C$ with a thyristor-switched $R, L$ branch in parallel in order to control the effective reactance of the device. In periodic steady state, in each half-period $T / 2$, the $R, L$ branch is connected over time $T_{1}$ and is interrupted over time $T_{2} \quad\left(T_{1}+T_{2}=T / 2\right)$. Thus, with $x_{1}=v_{C}$ and $x_{2}=i_{L}$ as state variables, over $T_{1}$ and $T_{2}$ the time-varying $A_{t}$ matrix of Section 2.2 becomes, respectively,

$$
A_{1}=\left[\begin{array}{c:c}
0 & -\frac{1}{C} \\
\frac{1}{L} & -\frac{R}{L}
\end{array}\right], \quad A_{2}=\left[\begin{array}{cc}
0 & -\frac{1}{C} \\
0 & 0
\end{array}\right]
$$

Thus the procedures of Section 2.2 for obtaining an LTI equivalent can be readily applied.

\subsection{Preview of Large Scale Studies}

Large scale (three-phase) studies must by necessity be modular. This applies to both the Harmonic Power Flow programs [2-13] and to the subsequent $s$-domain stability assessment. The latter entails the following steps:

(a) Formulation of $Y_{k}(s)$ for each component. These are readily available for transmission lines and lumped parameter elements. For nonlinear and time-varying components, LTI equivalents have to be produced, as shown in Section 2.2

(b) Assemble all $Y_{k}(s)$ into a system-wide $Y(s)$ matrix and obtain the values of $s$ for which $Y(s)$ becomes singular (see Section 2.1c).

\section{CONCLUSIONS}

The small signal stability analysis described in this paper is based on the fact that the linear, time-periodic (LTP) components, obtained by linearization (of both nonlinear and time-varying elements), can be replaced by linear, time-invariant (LTI, i.e. standard lumped parameter) components. The paper describes in detail this equivalencing procedure.

In most practical cases the system contains transmission lines, i.e. elements with frequency-dependent distributed parameters, which are best represented in the $s$-domain. Thus the $s$-domain methodology described in this study is sufficiently general for all practical situations. When, however, after equivalencing, the system can be modeled by linear, constant coefficient, differential equations, then the stability analysis can be formulated as a standard eigen-analysis problem. This is a simple, but less general, alternative to the $s$-domain methodology.

\section{ACKNOWLEDGMENTS}

Financial assistance by the Natural Sciences and Engineering Research Council of Canada is gratefully acknowledged

\section{REFERENCES}

[1] P.M. Anderson, B.L. Agrawal, and J.E. Van Ness, "Subsynchronous Resonance in Power Systems", IEEE Press, New York, 1990.

[2] A. Medina and J. Arrillaga, "Harmonic Interaction Between Generation and Transmission Systems", IEEE Transactions on Power Delivery, Vol, 8, October 1993 , pp. 1981-87.

[3] D. Xia and G.T. Heydt, "Harmonic Power Flow Studies Part l-Formulation and Solution", IEEE Transactions on Power Apparatus and Systems, Vol. PAS-101, No. 6, June 1982, pp. 1257-65.

[4] W. Xu, J.R. Marti, and HW. Dommel, "A Multiphase Harmonic Load Flow Solution Technique", IEEE Transactions on Power Systems, Vol. 6, No. 1, February 1991, pp. 174-82

[5] T.J. Demsem, P.S. Bodger, and J. Arrillaga, "Three Phase Transmission System for Harmonio Pepetration Studies", IEEE Transactions on Power Apparatus and Harmonio Penetration Studies", IEEE Transactions on

[6] E. Acha, J. Anrillaga, A. Medina, and A. Semlyen, "General Frame of Reference for Analysis of Harmonio Distortion in Systems with Multiple Transforme Nonlinearities", Proceedings IEE, Part C, Vol. 136, No. 5, September 1989, pp 271-78.

[7] H.W. Dommel, A. Yan, and S. Wei, "Harmonies from Transformer Saturation", IEEE Transactions on Power Systems, Vol. PWRD-1, No. 2, April 1986, pp. 20914.

[8] R. Yacamini and J.C. de Oliveira, "Harmonics in Multiple Convertor Systems: A Generalized Approach", Proceedings IEE Part B, Vol. 127, No. 2, March 1980, pp. 96-104.

[9] T.S. Parker and L.O. Chua, "Practical Numerical Algorithms for Chaotic Systems", Springer-Verlag, New Yotk, 1989

[10] J. Usaola and J.G. Mayordomo, "Fast Steady-State Technique for Harmonic Analysis", IEEE/ICHPS IV Fourth Intemational Conference on Hamonics in Power Systems, Budapest, October 4-6, 1990, pp. 33642.

[11] T.J. Aprille and T.N. Trick, "A Computer Algorithm to Determine the Steady-State Response of Nonlinear Oscillators", IEEE Transactions on Circuit Theory, Vol. CT-19, No. 4, July 1972 , pp. 354-60.

[12] A. Semlyen and A. Medina, "Computation of the Periodic Steady State in Systems with Nonlinear Components Using a Hybrid Time and Frequency Domain Methodology", IEEE Transactions on Power Systems, Vol. 10, August 1995, pp. 1498-1504.

[13] G.T. Heydt and J. Jun, "Rapid Calculation of the Periodic Steady State for Electronically Switched, Time Varying Power System Loads", IEEE Transactions on Power Delivery, Vol. 11, October 1996, pp. 1860-67.

[14] I. Dobson, "Stability of Ideal Thyristor and Diode Switching Circuits", IEEE Trartsactions on Circuits and Systems-1: Fundamental Theory and Applications, Vol. 42, No. 9, September 1995, pp. 517-529.

[15] I. Dobson, S.G. Jalali, and R. Rajaraman, "Damping and Resonance in a High Power I. Dobson, S.G. Jalali, and R. Rajaraman, "Damping and Resonance in a High Power Switching Circuit", pp. 137-156 in Systems and Control Theory for Power Systems, MA, Volume 64, Mathematics and its Applications, J.H,

[16] G. Floquet, "Sur les équations différentielles linéaires à coefficients périodiques", Annales Ecole Normale Supérieure, 12 , pp. 47-89.

[17] H. D'Angelo, 'Linear Time-Varying Systems: Analysis and Synthesis", Allyn and Bacon, Boston, 1970.

[18] M. Farkas, "Periodic Motions", Springer-Verlag, New York, 1994

[19] A. Semlyen and A. Deri, "Time Domain Modelling of Frequency Dependent ThreePhase Transmission Line Impedance", IEEE Transactions on Power Apparatus and Systems, Vol. PAS-104, June 1985, pp. 1549-55.

[20] A. Deri, G. Tevan, A. Semlyen, and A. Castanheira, "The Complex Ground Retum Plane: A Simplified Model for Homogeneous and Multi-Layer Earth Return", IFEE Transactions on Power Apparatus and Systems, Vol. PAS-100, August 1981, pp. 3686-93.

\section{BIOGRAPHY} Adam Semlyen was born in 1923 in Rumania where he obtained a Dipl. Ing. degree and
his Ph.D. He statted his career there with an electric power utility and held academic positions at the Polytechnic Institute of Timisoara. In 1969 he joined the University of positions at the Polytechnic Institute of Timisoara. In 1969 he joined the University of
Toronto where he is a professor in the Department of Electrical and computer engineering Toronto where he is a professor in the Department of Electrical and computer engineering,
emeritus since 1988 . His research interests include steady state and dynamic analysis as well as computation of electromagnetio transients in power systems. 